PSICOLOGÍA

IBEROAMERICANA
Psicología Iberoamericana ISSN: 1405-0943

revista.psicologia@ibero.mx

Universidad Iberoamericana, Ciudad de México México

\title{
Variables involucradas con el estrés académico y el afrontamiento en universitarios durante el confinamiento por covid-19
}

Avalos Latorre, María Luisa; Trujillo Martínez, Fanny

Variables involucradas con el estrés académico y el afrontamiento en universitarios durante el confinamiento por covid-19

Psicología Iberoamericana, vol. 29, núm. 3, Esp., e293331, 2021

Universidad Iberoamericana, Ciudad de México, México

Disponible en: https://www.redalyc.org/articulo.oa? id=133968747009

DOl: https://doi.org/10.48102/pi.v29i3.331

\section{(c) (1)}

Esta obra está bajo una Licencia Creative Commons Atribución 4.0 Internacional. 


\title{
Variables involucradas con el estrés académico y el afrontamiento en universitarios durante el confinamiento por covid-19
}

\author{
Variables involved with the academic stress and coping in \\ university students during COVID-19 confinement \\ María Luisa Avalos Latorre luisa.avalos@academicos.udg.mx \\ Universidad de Guadalajara, México \\ https://orcid.org/0000-0002-1183-1518 \\ Fanny Trujillo Martínez fanny.trujillo@uteg.edu.mx \\ Centro Universitario UTEG, México \\ https://orcid.org/0000-0003-0091-2010
} Psicología Iberoamericana, vol. 29, núm.
3, Esp., e293331, 2021

Universidad Iberoamericana, Ciudad de México, México

Recepción: 01 Febrero 2021 Aprobación: 18 Junio 2021

DOI: https://doi.org/10.48102/ pi.v29i3.331

Redalyc: https://www.redalyc.org/ articulo.oa?id $=133968747009$

Financiamiento

Fuente: Programa de Apoyo a la Mejora en las Condiciones de Producción SNI y SNCA (PROSNI) 2020.

No de contrato: Programa de Apoyo a la

Mejora en las Condiciones de Producción SNI y SNCA (PROSNI) 2020.

Beneficiario: María Luisa Avalos Latorre
Resumen: Se compararon los estresores académicos, la presencia de estrés académico y las estrategias de afrontamiento de estudiantes universitarios de acuerdo al sexo, el campo disciplinar y la disponibilidad tecnológica en tiempos de confinamiento por COVID 19 en la implementación de la enseñanza remota de emergencia. Mediante un estudio cuantitativo, transversal y comparativo, participaron 765 estudiantes universitarios de Jalisco, 568 mujeres y 197 hombres $(x \#=20.4$ años, $\min =17$, $\max =25)$, respondieron la Escala de Estresores Académicos, el Inventario de Estrés Académico SISCO, la Escala de Afrontamiento de Estrés Académico, y una ficha de datos demográficos y de disponibilidad tecnológica. Se realizó prueba $\mathrm{t}$ para muestras independientes, prueba ANOVA y estadísticos descriptivos. El 65.9\% de los participantes presentó nivel moderado de estrés. Se observaron diferencias estadísticamente significativas entre el nivel de estrés, el sexo $(\mathrm{t}=7.51, \mathrm{p}=.00)$ y el campo disciplinar $(\mathrm{F}=3.44$, $\mathrm{p}=.00)$, y diferencias significativas entre los dispositivos disponibles y los estresores $(\mathrm{F}=3.39, \mathrm{p}=.03)$. El estrés académico es un fenómeno complejo presente en población universitaria que debe abordarse desde las convencionalidades sociales, los campos disciplinares de las carreras universitarias y los estilos de afrontamiento delineados por la emergencia sanitaria.

Palabras clave: estrés académico, estrategias de afrontamiento, estudiantes universitarios, COVID-19.

Abstract: Academic stressors, the presence of academic stress and the coping strategies of university students were compared according to gender, disciplinary field and technological availability during times of confinement due to COVID-19 in the implementation of emergency remote teaching. Through a quantitative, cross-sectional and comparative study, 765 students participated. This included 568 women and 197 men $(x \#=20.4$ years, $\min =17, \max =25)$. The Scale of Academic Stressors, the SISCO Academic Stress Inventory, the Academic Stress Coping Scale, and demographic data and technological availability questions were tested on participants. A t-test was performed for independent samples. An ANOVA test and descriptive statistics were also used to analyse the data. Only $65.9 \%$ of the participants presented a moderate level of stress. Statistically significant differences were observed between stress level, gender $(\mathrm{t}=7.51, \mathrm{p}=.00)$ and disciplinary field $(\mathrm{F}=3.44, \mathrm{p}=.00)$, and significant differences between available devices and stressors $(\mathrm{F}=3.39, \mathrm{p}=.03)$. Academic stress is a complex phenomenon amongst the university population that must be approached from an 
understanding of social conventions, the disciplinary fields of different university careers and the coping styles outlined by the health emergency.

Keywords: academic stress, coping strategies, university students, COVID-19.

\section{Introducción}

En estos tiempos sin precedentes debido a la pandemia por COVID-19, el mundo entero se enfrenta a grandes retos, tal es el caso de la educación. Particularmente en México, el 28 de febrero del 2020, el Gobierno capitalino (INEE, 2019) confirmó al primer contagiado por COVID-19 en todo el país. Para el 20 de marzo del 2020 se cerraron las más de 243 480 escuelas de educación básica, media superior y superior que atendía a más de 30 millones de estudiantes, de acuerdo con el Instituto Nacional para la Evaluación de la Educación (INEE, 2019). Ello conllevó que las clases se llevaran a cabo de manera virtual, de tal forma que la apuesta de los servicios educativos resultó ser la estrategia de enseñanza remota de emergencia (Bozkurt \& Sharma, 2020; Niño et al., 2021). Para el sistema educativo esto implica un gran desafío, ya que México ocupa el lugar 76 de 140 países, en el índice de adopción de tecnologías de la información favorables al desarrollo económico (World Economic Forum, 2019).

El Instituto Nacional de Estadística y Geografía (INEGI, 2019), señala que en México en el 2019, la proporción total de hogares mexicanos que contaba con computadora fue de $44.3 \%$, así también, únicamente el $56.4 \%$ de hogares contaban con conexión a Internet y el $75.1 \%$ de la población de seis años en adelante eran usuarios de teléfono móvil, finalmente, señala que solo el $44.6 \%$ de las familias usaban la computadora como herramienta de apoyo escolar. Aunado a ello, Programa de Naciones Unidas para el Desarrollo (2020), afirma que dos de tres hogares de México no cuentan con una conexión de calidad a internet (velocidad suficiente), y solo en uno de cada tres hogares, la conexión se logra mediante una red fija de alta velocidad.

Estas dificultades tecnológicas han planteado dudas no sólo en torno a las barreras para el acceso a la educación sino también al impacto psicológico que estas condiciones pueden causar en los estudiantes. Por mencionar algunos estudios al respecto, González et al. (2020), evaluaron a 644 estudiantes universitarios mexicanos y observaron que una tercera parte presentó estrés, alteraciones de sueño y ansiedad. Por su parte, Jeff (2020), señaló que estudiantes de medicina de Perú al inicio de la pandemia mostraron síntomas ansiosos, estrés, alteraciones del sueño, disfunción social y depresión especialmente en mujeres y en los estudiantes universitarios más jóvenes. Asimismo, Ozamiz et al. (2020), señalaron que, en España, la población más joven mostró mayores niveles de estrés, depresión y ansiedad.

Aunque las alteraciones psicológicas tales como el estrés no son desconocidas en la población universitaria previo a la pandemia (Jayasankara et al., 2018; Macbani et al., 2018; Silva Ramos et al., 2019), la cancelación de las experiencias educativas prácticas, tales como asistir a laboratorios y comunidades, la realización del servicio social o el trabajo 
de investigación recepcional, e incluso el propio distanciamiento físico de los docentes y compañeros puede exacerbar los síntomas psicológicos o particularizarlos.

Chávez et al. (2019) definen al estrés académico como un proceso adaptativo y esencialmente psicológico, que ocurre cuando el alumno valora las demandas escolares como estresantes, llevándolo a una situación de estrés en el que experimentará indicadores del desequilibrio que lo obligarán a realizar acciones de afrontamiento para restaurar su equilibrio. Lazarus y Folkman (1986), explican este desequilibrio como una valoración de insuficientes recursos para hacer frente a unas demandas percibidas como desbordantes y amenazadoras.

Entre las demandas académicas más relevantes reportadas en investigaciones se encuentran los exámenes (González, Fernández et al., 2010; Vizoso Gómez \& Arias Gundín, 2016), la sobrecarga académica (Barraza et al., 2012; Toribio \& Franco, 2016), las intervenciones en público (González et al., 2014), y las deficiencias metodológicas del profesorado (Rodríguez et al., 2014).

Asimismo, los estudios sobre estrés académico publicados en los últimos años de distintas partes del mundo se han centrado principalmente en estudiantes de carreras pertenecientes a las ciencias de la salud dejando de lado otros campos disciplinares. Además, los estudios en donde se comparan los hombres y mujeres refieren una mayor vulnerabilidad del sexo femenino (Bedoya et al., 2014; Jerez \& Oyarzo, 2015; Román et al., 2008).

La manera en que un estudiante le hace frente a los estresores escolares son las estrategias de afrontamiento. Lazarus y Folkman (1986), las definen como los esfuerzos cognitivos y conductuales cambiantes que se desarrollan para manejar las demandas específicas externas y/o internas que son evaluadas como excedentes de los recursos del individuo.

Es así que un adecuado afrontamiento, por lo tanto, puede reducir el estrés y favorecer el bienestar psicológico del estudiante (Brougham et al., 2009; Fornés-Vives et al., 2016; Piemontesi et al., 2012).

La incertidumbre hacia lo que ocurrirá en los entornos educativos a nivel mundial por la pandemia exige un mayor conocimiento de las consecuencias que va dejando a su paso y los factores involucrados en ello.

Dado las pocas investigaciones que a la fecha han sido reportadas, resulta pertinente llevar a cabo estudios que permitan apreciar las condiciones y efectos que el confinamiento impone en la educación universitaria.

Además, es importante contrastar dichos efectos con hallazgos previos, lo cual nos permitirá proponer en un futuro estrategias efectivas y eficaces para adaptarnos óptimamente a la nueva normalidad.

Se parte de la hipótesis de que el sexo, el campo disciplinar y la disponibilidad tecnológica experimentan diferencias al comparar los estresores académicos, la presencia de estrés académico y las estrategias de afrontamiento de estudiantes universitarios. Por ello, el objetivo del presente trabajo fue comparar los estresores académicos, la presencia de estrés académico y las estrategias de afrontamiento de 
estudiantes universitarios de acuerdo con el sexo, el campo disciplinar y la disponibilidad tecnológica a ocho meses de confinamiento por COVID-19.

\section{Método}

Es un estudio de tipo cuantitativo, transversal y analítico. Se busca describir los resultados de las evaluaciones y comparar los estresores académicos, la presencia de estrés académico y las estrategias de afrontamiento de estudiantes universitarios de acuerdo con el sexo, el campo disciplinar y la disponibilidad tecnológica.

\section{Participantes}

Se incluyeron 765 estudiantes universitarios de dos Instituciones de Educación Superior de la ciudad de Guadalajara, Jalisco, México, el 73\% estaban adscritos a la Universidad de Guadalajara (UDG) y 27\% estaban adscritos a la Centro Universitario (UTEG). Asimismo, 568 (74\%) eran mujeres y 197 (26\%) hombres, tenían una edad promedio de 20.4 años (D.T. $=1.8, \min =17, \max =25$ ), el $97 \%$ eran solteros y el $3 \%$ casados. Respecto al campo disciplinar $74 \%$ estudiaban una carrera de ciencias de la salud, el 9\% estudiaban una carrera de las ciencias exactas, el 9\% estudiaban una carrera de las ciencias administrativas, el 6\% estudiaban carreras de las ciencias sociales y el $2 \%$ estudiaban carreras de diseño y arte.

El muestreo que se hizo fue por conveniencia, es decir, todos los jóvenes que aceptaron participar en el mes de noviembre y diciembre del 2020. Como criterios de inclusión se consideró que, al momento de recibir el formulario, estuvieran activos en alguna carrera universitaria y en cualquier semestre, que contaran con correo electrónico institucional y que aceptaran el consentimiento informado.

\section{Instrumentos}

Se empleó una ficha de datos elaborada previamente por los investigadores con preguntas cerradas y con opciones de respuesta en la que se recolectó la información sobre datos sociodemográficos, datos escolares y de disponibilidad tecnológica (acceso a internet, cantidad de dispositivos con el que cuenta, si comparte dispositivos en casa). Además de la ficha de datos se empleó la Escala de Estresores Académicos (ECEA; González et al., 2016), Inventario de Estrés Académico SISCO (Barraza, 2007) y la Escala de Afrontamiento de Estrés Académico (A-CEA; González, Valle et al., 2010), las propiedades psicométricas se describen a continuación.

\section{Escala de Estresores Académicos (ECEA)}

Consta de 54 reactivos que valorar el grado en que el estudiante universitario percibe situaciones o circunstancias del contexto académico 
que pueden ser percibidos como amenazantes. Considera ocho subescalas: deficiencias metodológicas del profesorado (DEFMET), sobrecarga académica del estudiante (SOBACA), creencias sobre el rendimiento académico (CRENREN), intervenciones en público (INTPUB), clima social negativo (CLINEG), exámenes (EXAM), creencia de valor de los contenidos (CARVAL), dificultades de participación (DIFPAR). Cada reactivo puntúa sobre una escala de estimación tipo Likert de 5 puntos ( 1 =nunca, $2=$ alguna vez, 3 =bastantes veces, $4=$ casi siempre, $5=$ siempre). Puntajes altos indican los estresores más relevantes percibidos por los estudiantes universitarios. La escala cuenta con una consistencia interna de un Alpha de Cronbach total de 0.96 en población peruana, asimismo, se valoró la validez de constructo por medio del análisis factorial exploratorio con el método de máxima verosimilitud obteniéndose un valor KMO de .874 (Chávez et al., 2019).

\section{Inventario de Estrés Académico SISCO}

Consta de 31 reactivos que permiten identificar la presencia y el nivel de estrés académico. Considera tres dimensiones: estresores (EST), reacciones (REAC) y estrategias de afrontamiento (ESTAFR). El primer ítem determina el nivel de preocupación o nerviosismo, ocho ítems identifican la frecuencia en que las demandas del entorno son valoradas como estímulos estresores, quince ítems identifican la frecuencia con que se presentan los síntomas o reacciones al estímulo estresor, mientras que siete ítems permiten identificar la frecuencia de uso de las estrategias de afrontamiento. Cada reactivo puntúa sobre una escala de estimación tipo Likert de 5 puntos ( $1=$ nunca, $2=$ alguna vez, $3=$ bastantes veces, $4=$ casi siempre, $5=$ siempre), la calificación del inventario es en orden sumativo, la cual se realiza por dimensiones y en totalidad ofreciendo tres niveles (alto, medio y bajo). La escala cuenta con una consistencia interna de un Alpha de Cronbach total de .82 en población mexicana, así también se realizó una prueba de esfericidad de Bartlett siendo significativa en .000, y el test KMO de adecuación de la muestra alcanzó un valor .762 (Barraza, 2007).

\section{Escala de Afrontamiento de Estrés Académico (A-CEA)}

Consta de 23 reactivos que permiten identificar las estrategias de afrontamiento del estrés utilizadas por los estudiantes en el ámbito universitario. Considera tres dimensiones: reevaluación positiva (REVPOS), búsqueda de apoyo (BUSAPO) y planificación (PLAN). Cada reactivo puntúa sobre una escala de estimación tipo Likert de 5 puntos $(1=$ nunca, $2=$ alguna vez, $3=$ bastantes veces, $4=$ casi siempre, $5=$ siempre), puntajes altos indican la predominancia determinada estrategia ello a través de tres niveles. La escala cuenta con una consistencia interna de un Alpha de Cronbach total de .89 en población española (Cabanach et al., 2010). 


\section{Procedimiento}

Los datos se obtuvieron mediante un formulario distribuido y aplicado en línea utilizando la aplicación Google Forms. Una vez que se localizaron los potenciales participantes a través de los docentes y de distintos directorios universitarios de las instituciones participantes, se les invitó a participar de manera voluntaria a través de Messenger, WhatsApp o e-mail. La encuesta estuvo disponible del 20 de noviembre al 10 de diciembre de 2020.

\section{Consideraciones éticas}

La presente investigación se sujetó a lo establecido en el Código Ético del Psicólogo Mexicano (Sociedad Mexicana de Psicología, 2007) y la Norma Oficial Mexicana NOM-012-SSA3-2012 (Secretaría de Gobernación, 2013), que establecen los criterios para la ejecución de proyectos de investigación para la salud en seres humanos. También se sujetó esta investigación al Código Helsinki (Asociación Médica Mundial, 2013). Se presentó a cada participante un consentimiento informado previo a su participación, el cual debía ser aceptado obligadamente para poder participar.

\section{Análisis de datos}

Los datos se analizaron y procesaron en el programa SPSS versión 24.0. Se realizaron pruebas $t$ para muestras independientes a datos que tuvieron distribución normal, para lo cual se aplicó previamente, la prueba de Kolmogorov-Smirnov, sí el valor fue mayor o igual a 0.05 existía normalidad y si fue menor la distribución fue no normal. También se realizó prueba ANOVA cuando se compararon tres o más variables.

\section{Resultados}

Los resultados respecto a la disponibilidad tecnológica indican que el $98 \%$ de los estudiantes sí cuenta con internet en casa, por otro parte el 54\% afirmó que comparte los dispositivos con otros integrantes de su familia. El 80\% de los participantes en este estudio contaba con computadora y teléfono móvil, el resto contaba sólo con alguno de ellos o bien con una Tablet.

En cuanto a los resultados obtenidos por cada una de las escalas, en la Tabla 1 se describen los resultados referentes a los estresores académicos, los niveles de estrés y las estrategias de afrontamiento. La evaluación de los estresores académicos mostró un mayor porcentaje en el nivel medio y alto en la dimensión deficiencias metodológicas del profesorado ( $40.5 \%$ y $25.2 \%$ respectivamente) y sobrecarga académica (36.3\% y $28.1 \%$ respectivamente). Con relación a los niveles de estrés, la mayoría de los participantes reportó un nivel medio de estrés (65.9\%). Finalmente, en las 
estrategias de afrontamiento la dimensión planificación mostró un mayor porcentaje en el nivel medio (47.8\%) y en el nivel alto (20.1\%).

Tabla 1

Tabla 1

Resultados porcentuales de las escalas sobre estresores académicos, nivel de estrés y estrategias de afrontamiento

\begin{tabular}{|c|c|c|c|c|}
\hline \multirow{2}{*}{ Variables } & \multirow{2}{*}{ Dimensiones } & \multicolumn{3}{|c|}{ Niveles } \\
\hline & & Bajo & Medio & Alto \\
\hline \multirow{9}{*}{$\begin{array}{l}\text { Estresores } \\
\text { Académicos }\end{array}$} & Deficiencias metodológicas del profesorado & 34.2 & 40.5 & 25.2 \\
\hline & Sobrecarga académica del estudiante & 35.6 & 36.3 & 28.1 \\
\hline & Creencias sobre el rendimiento académico & 42.5 & 32.3 & 25.2 \\
\hline & Intervenciones en público & 53.2 & 30.1 & 16.7 \\
\hline & Clima social negativo & 62.4 & 26.3 & 11.4 \\
\hline & Exámenes & 44.4 & 33.2 & 22.4 \\
\hline & Creencia de valor de los contenidos & 66.0 & 23.1 & 10.8 \\
\hline & Dificultades de participación & 54.5 & 27.5 & 18.0 \\
\hline & GLOBAL & 42.9 & 42.0 & 15.2 \\
\hline & Estresores & 15.4 & 55.0 & 29.5 \\
\hline Estrés & Reacciones & 17.9 & 52.7 & 29.4 \\
\hline \multirow[t]{3}{*}{ Académico } & Estrategias de afrontamiento & 19.6 & 62.5 & 17.9 \\
\hline & GLOBAL & 12.9 & 65.9 & 21.2 \\
\hline & Reevaluación positiva & 28.9 & 48.2 & 22.9 \\
\hline \multirow{3}{*}{$\begin{array}{l}\text { Estrategias de } \\
\text { afrontamiento }\end{array}$} & Búsqueda de apoyo & 28.4 & 42.9 & 28.8 \\
\hline & Planificación & 21.0 & 47.8 & 31.1 \\
\hline & GLOBAL & 24.8 & 55.0 & 20.1 \\
\hline
\end{tabular}

$\mathrm{n}=765$. Nota. los datos se presentan en porcentajes.

$\mathrm{n}=765$. Nota. los datos se presentan en porcentajes.

\section{Comparación por sexo}

Mediante la prueba t para muestras independientes, se encontraron diferencias significativas positivas entre el sexo y las dos dimensiones de estresores académicos: intervenciones en público $(\mathrm{t}=6.49, \mathrm{p}=.00)$ y clima social negativo $(\mathrm{t}=3.54, \mathrm{p}=.00)$. También se identificaron diferencias significativas positivas del sexo con el nivel de estrés $(t=7.20, p=.00)$ y la dimensión reacciones $(\mathrm{t}=7.20, \mathrm{p}=.00)$. Finalmente, se observó diferencia significativa negativa entre el sexo y la estrategia de afrontamiento reevaluación positiva $(\mathrm{t}=-3.20, \mathrm{p}=.00)($ Ver Tabla 2$)$. 
Tabla 2

Tabla 2

Comparación de estresores académicos, nivel de estrés y estrategias de afrontamiento según sexo

\begin{tabular}{|c|c|c|c|c|c|c|}
\hline \multirow{2}{*}{ Var. } & \multirow{2}{*}{ Dim. } & \multicolumn{2}{|c|}{ Mujeres } & \multicolumn{2}{|c|}{ Hombres } & \multirow[t]{2}{*}{$t$} \\
\hline & & M & D.E. & $M$ & D.E. & \\
\hline \multirow[t]{9}{*}{$T$} & DEFMET & 35.79 & 11.79 & 30.12 & 12.28 & 5.75 \\
\hline & SOBACA & 29.97 & 10.82 & 24.05 & 10.54 & 6.66 \\
\hline & CRENREN & 28.28 & 10.88 & 23.28 & 10.63 & 5.59 \\
\hline & INTPUB & 13.33 & 5.54 & 10.44 & 4.91 & $6.49^{*}$ \\
\hline & CLINEG & 13.68 & 6.20 & 11.92 & 5.46 & $3.54^{*}$ \\
\hline & EXAM & 11.47 & 4.38 & 9.20 & 4.02 & 6.40 \\
\hline & CARVAL & 9.26 & 4.07 & 8.27 & 3.83 & 2.96 \\
\hline & DIFPAR & 7.27 & 3.36 & 6.24 & 3.17 & 3.76 \\
\hline & GLOBAL & 149.05 & 47.18 & 123.53 & 46.57 & 6.56 \\
\hline \multirow[t]{4}{*}{ II } & EST & 29.05 & 6.80 & 25.35 & 7.51 & 6.40 \\
\hline & REAC & 49.42 & 12.40 & 41.72 & 14.39 & $7.20^{*}$ \\
\hline & ESTAFR & 20.18 & 4.91 & 19.31 & 5.35 & 2.07 \\
\hline & GLOBAL & 98.64 & 18.69 & 86.38 & 22.46 & $7.52^{*}$ \\
\hline \multirow[t]{4}{*}{ IIII } & REVPOS & 25.56 & 7.50 & 27.62 & 8.59 & $-3.20^{\star}$ \\
\hline & BUSAPO & 20.58 & 6.74 & 19.85 & 7.22 & 1.28 \\
\hline & PLAN & 21.14 & 6.23 & 21.52 & 6.89 & -.71 \\
\hline & GLOBAL & 67.28 & 18.12 & 68.98 & 20.59 & -1.10 \\
\hline \multicolumn{7}{|c|}{ 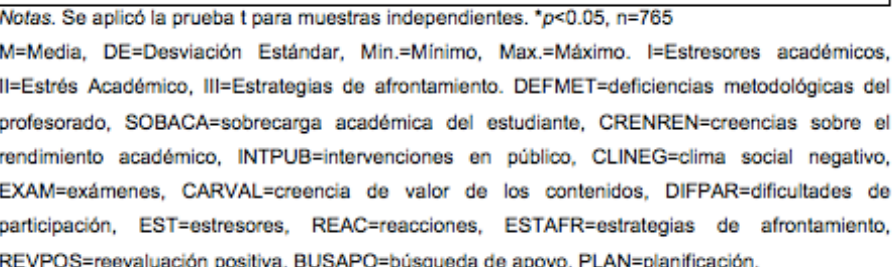 } \\
\hline
\end{tabular}

Notas. Se aplicó la prueba t para muestras independientes. ${ }^{*} \mathrm{p}<0.05, \mathrm{n}=765$

$\mathrm{M}=$ Media, DE=Desviación Estándar, Min.=Mínimo, Max.=Máximo. I=Estresores académicos, II=Estrés Académico, $\mathrm{III}=$ Estrategias de afrontamiento. $\mathrm{DEFMET}=$ deficiencias metodológicas del profesorado, $\mathrm{SOBACA}=$ sobrecarga académica del estudiante, $\mathrm{CRENREN}=$ creencias sobre el rendimiento académico, INTPUB=intervenciones en público, $C L I N E G=$ clima social negativo, $E X A M=$ exámenes, $C A R V A L=$ creencia de valor de los contenidos, DIFPAR=dificultades de participación, EST=estresores, $R E A C=$ reacciones, $E S T A F R=$ estrategias de afrontamiento, $\mathrm{REVPOS}=$ reevaluación positiva, $\mathrm{BUSAPO}=$ búsqueda de apoyo, $\mathrm{PLAN}=$ planificación.

\section{Comparación por campo disciplinar}

Al comparar el campo disciplinar (Cs. de la salud, Cs. Exactas, Cs. Administrativas, Cs. Sociales, Diseño y Arte) de acuerdo con los estresores académicos, se observaron significancias estadísticas con las dimensiones: deficiencias metodológicas del profesorado $(\mathrm{F}=3.86, .=.00)$, sobrecarga académica del estudiante $(\mathrm{F}=4.76, \mathrm{p}=.00)$, creencias sobre el rendimiento académico $(\mathrm{F}=3.27, \mathrm{p}=.01)$, intervenciones en público $(\mathrm{F}=5.13, \mathrm{p}=.00)$, exámenes $(\mathrm{F}=5.83, \mathrm{p}=.00)$ y afrontamiento global $(\mathrm{F}=4.27, \mathrm{p}=.00)$. También se observó diferencia significativa entre el campo disciplinar, el nivel de estrés $(\mathrm{F}=3.44, \mathrm{p}=.00)$ y la dimensión reacciones del inventario SISCO ( $\mathrm{F}=3.51, \mathrm{p}=.00)$. No observamos diferencias significativas entre el campo disciplinar y las estrategias de afrontamiento (Ver Tabla 3). 
Tabla 3

Tabla 3

\begin{tabular}{|c|c|c|c|c|c|}
\hline Var. & Dim. & M & $\mathrm{DE}$ & Min-Max & $F$ \\
\hline \multirow{9}{*}{ I } & DEFMET & 34.33 & 12.16 & $12-60$ & $3.86^{*}$ \\
\hline & SOBACA & 28.45 & 11.05 & $10-50$ & $4.76^{*}$ \\
\hline & CRENREN & 26.99 & 11.03 & $10-50$ & $3.26^{*}$ \\
\hline & INTPUB & 12.59 & 5.53 & $5-25$ & $5.13^{*}$ \\
\hline & CLINEG & 13.22 & 6.06 & $6-30$ & .567 \\
\hline & EXAM & 10.88 & 4.40 & $4-20$ & $5.82^{*}$ \\
\hline & CARVAL & 9.00 & 4.03 & $4-20$ & .636 \\
\hline & DIFPAR & 7.01 & 3.35 & $3-15$ & 1.71 \\
\hline & GLOBAL & 142.48 & 48.30 & $54-270$ & $4.27^{*}$ \\
\hline \multirow{4}{*}{ II } & EST & 28.10 & 7.17 & $11-43$ & 1.32 \\
\hline & REAC & 47.43 & 13.36 & $15-75$ & $3.50^{*}$ \\
\hline & ESTAFR & 19.96 & 5.04 & $9-33$ & 1.36 \\
\hline & GLOBAL & 95.49 & 20.43 & $35-151$ & $3.44^{*}$ \\
\hline \multirow{4}{*}{ III } & REVPOS & 26.09 & 7.84 & $9-45$ & .281 \\
\hline & BUSAPO & 20.39 & 6.87 & $7-35$ & .255 \\
\hline & PLAN & 21.24 & 6.40 & $7-35$ & .304 \\
\hline & GLOBAL & 67.72 & 18.79 & $23-115$ & .202 \\
\hline \multicolumn{6}{|c|}{$\begin{array}{l}\text { M=Media, DE-Desviación Estándar, Min.=Minimo, Max.=Máximo. I-Estresores académicos, II=Estrés } \\
\text { Académico, III=Estrategias de afrontamiento. DEFMET=deficiencias metodológicas del profesondo, } \\
\text { SOBACA=sobrecarga académica del estudiante, CRENREN=creencias sobre el rendimiento académico, } \\
\text { INTPUB-intervenciones en público, CLINEG=clima social negativo, EXAM-exámenes, CARVAL=creencia de } \\
\text { valor de los contenidos, DIFPAR=dificultades de participación, EST-estresores, REAC-reacciones, } \\
\text { ESTAFR=estrategias de afrontamiento, REVPOS=recvaluación positiva, BUSAPO=búsqueda de apoyo, } \\
\text { PLAN-planificación. }\end{array}$} \\
\hline
\end{tabular}

Notas. Se aplicó la prueba ANOVA de un factor. ${ }^{*} \mathrm{p}<0.05, \mathrm{n}=765$

M=Media, DE=Desviación Estándar, Min.=Mínimo, Max.=Máximo. I=Estresores académicos, II=Estrés Académico, $\mathrm{III}=$ Estrategias de afrontamiento. DEFMET =deficiencias metodológicas del profesorado, $\mathrm{SOBACA}=$ sobrecarga académica del estudiante, $\mathrm{CRENREN}=$ creencias sobre el rendimiento académico, INTPUB=intervenciones en público, CLINEG=clima social negativo, EXAM=exámenes, $\mathrm{CARVAL}=$ creencia de valor de los contenidos, DIFPAR=dificultades de participación, EST=estresores, REAC=reacciones, ESTAFR=estrategias de afrontamiento, REVPOS=reevaluación positiva, $\mathrm{BUSAPO}=$ búsqueda de apoyo, $\mathrm{PLAN}=$ planificación.

Para identificar entre qué categorías había diferencias, llevamos a cabo una prueba post hoc de Tukey, se observó significancia en la dimensión deficiencias metodológicas entre los estudiantes de ciencias de la salud y los estudiantes de ciencias administrativas $(\mathrm{p}=.00)$. En la dimensión sobrecarga académica del estudiante se observó significancia entre los estudiantes de ciencia de la salud y los estudiantes de ciencias administrativas.

En la dimensión intervenciones en público, se observaron significancias entre estudiantes de ciencias de la salud y ciencias sociales $(\mathrm{p}=.04)$, entre ciencias de la salud y ciencias administrativas $(\mathrm{p}=.03)$, y entre ciencias de la salud y ciencias exactas $(\mathrm{p}=.04)$. En la dimensión exámenes, se identificaron significancia entre estudiantes de ciencias de la salud y ciencias sociales $(\mathrm{p}=.03)$, y entre estudiantes de ciencias de la salud y ciencias administrativas $(\mathrm{p}=.00)$. 
Aunado a ello, el puntaje global de estresores académicos mostró significancia entre los estudiantes de ciencias de la salud y los de ciencias administrativas $(\mathrm{p}=.02)$. Finalmente, el nivel de estrés mostró diferencias significativas entre ciencias de la salud y ciencias sociales $(p=.04)$.

\section{Comparación por disponibilidad de tecnología}

Enseguida, se compararon las variables de estudio con los indicadores de disponibilidad tecnológica. No se identificaron diferencias significativas entre contar internet en casa, los estresores académicos, la presencia de estrés y las estrategias de afrontamiento. La cantidad de dispositivos mostró diferencias significativas con las dimensiones sobrecarga académica $(\mathrm{F}=3.24, \mathrm{p}=.04)$, dificultades de participación $(\mathrm{F}=2.85$, $\mathrm{p}=.05)$ y la percepción global de estresores académicos $(\mathrm{F}=3.39$, $\mathrm{p}=.03)$. Respecto a compartir dispositivos no se observaron diferencias estadísticamente significativas con las estrategias de afrontamiento (Ver Tabla 4).

\section{Tabla 4}

Tabla 4

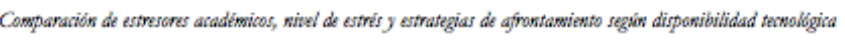

\begin{tabular}{|lccccccccc|}
\hline \multirow{2}{*}{ Dim. } & \multicolumn{3}{c}{ Internet en casa } & \multicolumn{3}{c}{ Cantidad de dispositivos } & \multicolumn{3}{c|}{ Compartir dispositivo } \\
\cline { 2 - 10 } & $\mathrm{M}$ & $\mathrm{DE}$ & $t$ & $\mathrm{M}$ & $\mathrm{DE}$ & $F$ & $\mathrm{M}$ & $\mathrm{DE}$ & $t$ \\
\hline DEFMET & 34.36 & 12.17 & .43 & 34.33 & 12.16 & 2.79 & 34.08 & 12.18 & -.62 \\
\hline SOBACA & 28.42 & 11.04 & -.45 & 28.45 & 11.05 & $3.24^{*}$ & 28.41 & 11.13 & -.09 \\
\hline CRENREN & 27 & 11.08 & .11 & 26.99 & 11.03 & 2.65 & 26.89 & 11.01 & -.28 \\
\hline INTPUB & 12.6 & 5.56 & .53 & 12.59 & 5.53 & 2.40 & 12.78 & 5.45 & 1.04 \\
\hline CLINEG & 13.2 & 6.06 & -.77 & 13.22 & 6.06 & 2.36 & 13.28 & 6.07 & .27 \\
\hline EXAM & 10.88 & 4.4 & -.22 & 10.88 & 4.4 & 2.16 & 11.04 & 4.35 & 1.04 \\
\hline CARVAL & 8.98 & 4.04 & -.91 & 9 & 4.03 & .32 & 8.92 & 4.03 & -.61 \\
\hline DIFPAR & 6.98 & 3.34 & -1.53 & 7.01 & 3.35 & $2.85^{*}$ & 7.1 & 3.39 & .79 \\
\hline GLOBAL & 142.42 & 48.42 & -.21 & 142.48 & 48.3 & $3.39 *$ & 142.49 & 48.75 & .01 \\
\hline EST & 28.09 & 7.19 & -.04 & 28.1 & 7.17 & 2.05 & 28.27 & 7.08 & .74 \\
\hline REAC & 47.35 & 13.39 & -1.15 & 47.43 & 13.36 & 1.28 & 47.64 & 13.07 & .47 \\
\hline ESTAFR & 19.96 & 5.04 & .06 & 19.96 & 5.04 & 1.99 & 20.11 & 5.12 & .91 \\
\hline GLOBAL & 95.4 & 20.45 & -.74 & 95.49 & 20.43 & 1.61 & 96.02 & 20.37 & .79 \\
\hline REVPOS & 26.12 & 7.87 & .73 & 26.09 & 7.84 & .93 & 26.06 & 7.7 & -.12 \\
\hline BUSAPO & 20.43 & 6.9 & 1.20 & 20.39 & 6.87 & 1.35 & 20.33 & 6.6 & -.25 \\
\hline PLAN & 21.24 & 6.4 & .08 & 21.24 & 6.4 & 1.83 & 21.15 & 6.24 & -.42 \\
\hline GLOBAL & 67.8 & 18.85 & .77 & 67.72 & 18.79 & 1.50 & 67.54 & 18.58 & -.28 \\
\hline
\end{tabular}

M=Media, DE=Desviación Estándar, Min.=Minimo, Max.=Máximo. I=Estresores académicos, II-Estrés Académico, III-Estrategias de afrontamiento. DEFMET-deficiencias metodológicas del profesorado, SOBACA=sobrecarga académica del estudiante, CRENREN=creencias sobre el rendimiento académico, INTPUB=intervenciones en público, CLINEG=clima social negativo, EXAM-exámenes, CARVAL-creencia de valor de los contenidos, DIFPAR=dificultades de participación, EST=estresores, REAC-reacciones, ESTAFR=estrategias de afrontamiento, REVPOS=reevaluación positiva, BUSAPO=búsqueda de apoyo, PLAN=planificación.

M=Media, DE=Desviación Estándar, Min.=Mínimo, Max.=Máximo. I=Estresores académicos, II=Estrés Académico, $\mathrm{III}=$ Estrategias de afrontamiento. $\mathrm{DEFMET}=$ deficiencias metodológicas del profesorado, $\mathrm{SOBACA}=$ sobrecarga académica del estudiante, CRENREN=creencias sobre el rendimiento académico, INTPUB=intervenciones en público, $\mathrm{CLINEG=clima} \mathrm{social} \mathrm{negativo,} \mathrm{EXAM=exámenes,} \mathrm{CARVAL}=$ creencia de valor de los contenidos, 
DIFPAR=dificultades de participación, EST=estresores, $\mathrm{REAC}=$ reacciones, $\mathrm{ESTAFR}=$ estrategias de afrontamiento, REVPOS=reevaluación positiva, BUSAPO=búsqueda de apoyo, PLAN=planificación.

Por último, los resultados obtenidos con la prueba ANOVA, entre el nivel de estrés y las estrategias de afrontamiento, señalan diferencias significativas entre el nivel de estrés y la estrategia de búsqueda de apoyo $(\mathrm{F}=3.97, \mathrm{p}=.019)$. La prueba post hoc de Tukey arrojó significancia entre el nivel bajo de estrés y la estrategia de afrontamiento búsqueda de apoyo $(\mathrm{p}=.036)$.

\section{Discusión}

La hipótesis planteada en esta investigación fue que el sexo, el campo disciplinar y la disponibilidad tecnológica mostraban diferencias al comparar los estresores académicos, la presencia de estrés académico y las estrategias de afrontamiento de estudiantes universitarios.

Más de la mitad de los estudiantes universitarios participantes en el estudio presentaron un nivel de estrés moderado, estos hallazgos coinciden con otras investigaciones en los que, estudiantes universitarios, reportaron la presencia de estrés académico (Jerez \& Oryazo, 2015; Silva Ramos et al., 2019; Toribio \& Franco, 2016), la cual, además, es un padecimiento que se ha hecho aún más presente en estos tiempos de confinamiento por la pandemia COVID-19 (González et al., 2020; Jeff, 2020). Estos hallazgos parecen indicar que las condiciones educativas remotas debidas a la pandemia enmarcan un contexto en donde se hacen presentes estímulos que le resultan estresantes a los estudiantes universitarios. Quezada y González (2012), señalan que el proceso del estrés comienza cuando un individuo se enfrenta a estresores, que se valoran como desbordantes a los recursos, donde además existe una afectación a la calidad de vida del individuo.

Además, tal como ha sido reportado por otros autores (Bedoya et al., 2014; Román et al., 2008), las mujeres universitarias son más vulnerables al estrés y a las reacciones físicas, psicológicas y comportamentales que éste conlleva. Esta evidencia lleva a la necesidad de considerar también una perspectiva sociocultural del estrés tal y como Segura y Pérez (2016) lo señalan, ya que, los estresores pueden ser similares para hombres y mujeres, pero en la forma en cómo son valorados, como se reacciona ante ellos, cómo afectan y cómo se afrontan se involucran atributos propiamente convencionales históricos.

Aunado a ello, las diferencias de género socialmente establecidas para mujeres, tienen una repercusión importante en el resultado de estrés. Esto debido a los roles de trabajo doméstico y crianza de los hijos con el que se educa a las mujeres aun cuando se encuentren en situaciones de desarrollo profesional (Chávez, 2004; Montesó, 2014; Reverter Bañón, 2003).

Lo anterior nos ayuda a entender esa diferencia encontrada entre sexos, en estos tiempos de pandemia, que además se multiplica con el aumento en las cargas de trabajo no remuneradas de las mujeres. 
Entre dicho trabajo se puede señalar el mayor tiempo que tienen que pasar en casa por el confinamiento, la pérdida de puestos de trabajo, el cierre de escuelas y la enfermedad contraída de sus dependientes.

El estrés académico se experimenta de manera diferente en los estudiantes de carreras de ciencias de la salud, se muestran más afectados, si bien es cierto que el estrés está presente en la mayoría de los estudiantes, el contacto con la enfermedad, el sufrimiento y la muerte aunado a la exigencia académica son aspectos que afectan el modo de percibir el estrés (Bedoya et al., 2014; de La Rosa et al., 2015; del Toro et al., 2011).

Valdrá la pena revisar si los niveles de estrés identificados en estudiantes que estudian carreras de ciencias de la salud son de tipo crónico debido a que los estresores a los que están expuestos son permanentes o habituales o simplemente es un fenómeno que acontece y se exacerba por las condiciones ambientales propias de la pandemia.

La disponibilidad tecnológica (disponer de internet en casa, cantidad de dispositivos y compartir dispositivos con otros integrantes de la familia) no fue una variable que diferenció al estrés académico, Herrera (2009), señala que los jóvenes universitarios en México adoptan los recursos tecnológicos a las actividades académicas desde ya hace mucho tiempo. Aunado a ello, la cantidad de equipos disponibles por los estudiantes marcó diferencias entre los estresores percibidos, Alcibar et al. (2018), también afirman que los estudiantes en México utilizan diversas herramientas informáticas y se sienten más complacidos cuando cuentan con equipos tecnológicamente amigables y con computadora, ello puede hacer sentir al estudiante con mayor seguridad al contar con recursos suficientes para hacerle frente a las exigencias y desafíos escolares.

En esta investigación la disponibilidad tecnológica no diferenció al estrés por lo que, las fuentes de estrés pueden más bien ser derivadas de las debilidades de la enseñanza remota de emergencia y la escasa preparación de los docentes para afrontar las exigencias educativas en tiempos de pandemia. Este hallazgo también se encontró en investigaciones realizadas en España (Fernández-Regueira et al., 2020) y México (Portillo Peñuelas et al., 2020).

Aunque habría que realizar estudios con mayor profundidad al respecto, los hallazgos respecto a los estresores percibidos por los estudiantes universitarios coinciden con aspectos propios de la enseñanza: tal es el caso de las deficiencias metodológicas del profesorado y sobrecarga académica.

Respecto a las estrategias de afrontamiento identificadas destacan la reevaluación positiva únicamente al comparar por sexo, siendo los hombres los que refieren crear un nuevo significado de la situación del problema, tratando de resaltar los aspectos positivos o activando expectativas positivas. Este hallazgo discrepa de otros estudios en los cuales se reporta que las mujeres tienden a emplear estrategias de afrontamiento más efectivas (Martín et al., 2011, Reyes et al., 2017). Suponemos que la emergencia del coronavirus delinea acciones personales para hacerle frente a los desafíos del día a día, en el que, las mujeres están en mayor desventaja tal como lo mencionamos en líneas anteriores. 
Una de las debilidades del estudio tiene que ver con la forma en que se recolectaron los datos, ya que, al ser en línea a través de la invitación por correo electrónico o por mensajería instantánea, casi toda la muestra tenía fácil acceso a internet. Debido a esto, resultará importante que en futuros estudios se localicen presencialmente a aquellos estudiantes que no cuenten con él y así contrastar los hallazgos con estudiantes que requieren salir de su hogar para conectarse a sus cursos universitarios. Ello una vez que a un año de iniciar la pandemia, existen claras medidas de seguridad e higiene para prevenir contagios y el proceso de vacunación de la población ha comenzado.

Una visión global del estrés, como es el caso de esta investigación, permite identificar la importancia de las condiciones del proceso de enseñanza-aprendizaje tales como las relaciones sociales, la metodología de enseñanza, el campo disciplinar, así como las características personales de los estudiantes universitarios y el uso de la tecnología como medio para la enseñanza remota emergente. Por ello, resultará importante examinar si las carreras propias de las ciencias de la salud son estresantes por sí mismas o lo son debido a las prácticas de enseñanza arraigadas y a los estereotipos culturales que se tiene de ellas.

El estrés puede llegar a afectar la calidad de vida de las personas y su estado de salud y llevar a problemas de salud graves tanto en el plano físico como psicológico (Lazarus \& Folkman, 1986). Los resultados de la presente investigación dejan entrever la importancia de implementar programas de intervención psicológica, para que el estudiante universitario adquiera estrategias de autocontrol en el uso eficiente de hábitos de estudio (Fox, 1962; Olivares et al., 2014) y técnicas de programación conductual acompañadas de técnicas de relajación y respiración (Méndez et al., 2014). Se esperaría que con dicho entrenamiento se posibiliten mejores hábitos de estudio y autogestión. Finalmente, valdrá la pena continuar profundizando en el estudio del impacto que la enseñanza remota emergente y la disponibilidad tecnológica traerá en la calidad educativa y en el bienestar psicológico de los estudiantes.

\section{Conclusión}

El estrés académico se presentó en la mayoría de los estudiantes universitarios evaluados siendo las mujeres las mayormente afectadas, así como los estudiantes de carreras de ciencias de la salud. Los estímulos escolares percibidos con mayor frecuencia como estresantes tienen que ver con las estrategias de enseñanza. Aunado a ello, la cantidad de dispositivos para el acceso a las clases remotas mostró ser un indicador importante para contrarrestar el estrés más que el acceso a internet y la calidad de éste.

Para contrarrestar la presencia de estrés en estudiantes universitarios en tiempos de pandemia, se requiere abordar el fenómeno desde una perspectiva socio-cultural y centrado en las estrategias de enseñanza acordes con la virtualidad así como con pertinencia en los conocimientos, habilidades y actitudes genéricos y particulares de cada campo disciplinar. 


\section{Agradecimientos}

Fuente de financiamiento: Programa de Apoyo a la Mejora en las Condiciones de Producción SNI y SNCA (PROSNI) 2020.

\section{Referencias}

Alcibar, M., Monroy, A., \& Jiménez, M. (2018). Impacto y aprovechamiento de las tecnologías de la información y las comunicaciones en la educación superior. Información Tecnológica, 29(5), 101-110. http://dx.doi.org/10. 4067/S0718-07642018000500101.

Asociación Médica Mundial (AMM). (2013). Declaración de Helsinki de la AMM - Principios éticos para las investigaciones médicas en seres humanos. https://www.wma.net/es/policies-post/declaracion-de-helsink i-de-la-amm-principios-eticos-para-las-investigaciones-medicas-en-sereshumanos/

Barraza, A. (2007). Propiedades psicométricas del Inventario SISCO del estrés académico. Revista Psicología Cientifica, 7, 89-93.

Barraza, A., Martínez, J., Silva, J., Camargo, E., \& Antuna, R. (2012). Estresores académicos y género. Un estudio exploratorio de su relación en alumnos de licenciatura. Visión Educativa IUNAES, 5(12), 33-43.

Bedoya, F., Matos, L., \& Zelaya, E. (2014). Niveles de estrés académico, manifestaciones psicosomáticas y estrategias de afrontamiento en alumnos de la facultad de medicina de una universidad privada de Lima en el año 2012. Revista de Neuropsiquiatria, 77(4), 262-270.

Bozkurt, A., \& Sharma, R. (2020). Emergency remote teaching in a time of global crisis due to Coronavirus pandemic. Asian Journal of Distance Education, 15(1).

Brougham, R., Zail, C., Mendoza, C., \& Miller, J. (2009). Stress, sex differences, and coping strategies among college students. Current Psychology, 28, 85-97. https://doi.org/10.1007/s12144-009-9047-0

Cabanach, R., Valle, A., Rodríguez, S., Piñeiro, I., \& Freire, C. (2010). Escala de afrontamiento del estrés académico (A-CEA). Revista Iberoamericana de Psicologia y Salud, 1(1), 51-64.

Chávez, C. J. (2004). Perspectiva de Género. Editorial Plaza Valdez \& UNAM.

Chávez, T., Cuadros, M., López, A., Montoya, M., Requena, N., \& Talavera, Y. (2019). Análisis psicométrico de la Escala de Estresores Académicos (ECEA), en estudiantes de la Universidad Católica de Santa María. Avances en Psicologia, 27(1), 73-82. https://doi.org/10.33539/avpsicol.2 019v27n1.1467.

De La Rosa, G., Chang, S., Delgado, L., Oliveros, L., Murillo, D., Ortiz, R., Vela, G., \& Carreazo, N. Y. (2015). Niveles de estrés y formas de afrontamiento en estudiantes de Medicina en comparación con estudiantes de otras escuelas. Gaceta Médica de México, 151(4), 443-449.

Del Toro, Y., Gorguet, M., Pérez, Y., \& Ramos, D. (2011). Estrés académico en estudiantes de medicina de primer año con bajo rendimiento escolar. MEDISAN, 15(1), 17-22. 
Fernández-Regueira, U., Gewerc, A., \& Llamas-Nistal, M. (2020). El profesorado universitario de galicia y la enseñanza remota de emergencia: condiciones y contradicciones. Campus Virtuales, 9(2), 9-24.

Fornés-Vives, J., Garcia-Banda, G., Frias-Navarro, D., \& Rosales-Viladrich, G. (2016). Coping, stress, and personality in Spanish nursing students: A longitudinal study. Nurse Education Today, 36, 318-323. https://doi.org /10.1016/j.nedt.2015.08.011

Fox, L. (1962). Effecting the use of efficient study habits. Journal of Mathetics, I(1), 75-86.

González, C., Fernández, C., González, D., \& Freire, R. (2010). Estresores académicos percibidos por estudiantes universitarios de ciencias de la salud. Fisioterapia, 32(4), 151-158. https://doi.org/10.1016/j.ft.2010.01 .005

González, C., Souto, A., \& Franco, V. (2016). Escala de Estresores Académicos para la evaluación de los estresores académicos en estudiantes universitarios. Revista Iberoamericana de Psicologia y Salud, 7, 41-50. http ://dx.doi.org/10.1016/j.rips.2016.05.001.

González, N., Tejeda, A., Espinosa, C., \& Ontiveros, Z. (2020). Impacto psicológico en estudiantes universitarios mexicanos por confinamiento durante la pandemia por Covid-19. Scielo Preprints. https://doi.org/10.1 590/SciELOPreprints.756.

González, R., Souto, A., Freire, C., \& Ferradás, M. (2014). Relaciones entre autoestima y estresores percibidos en estudiantes universitarios. European Journal of Education and Psychology, 7(1), 43-57. https://doi.org/10.198 9/ejep.v7i1.151.

González, R., Valle, A., Rodríguez, S., Piñero, I., \& Freire C. (2010). Escala de Afrontamiento del Estrés Académico (A-CEA). Revista Iberoamericana de Psicología y Salud, 1(1), 51-64.

Herrera, M. (2009). Disponibilidad, uso y apropiación de las tecnologías por estudiantes universitarios en México: perspectivas para una incorporación innovadora. Revista Iberoamericana de Educación, 48(6), 1-9.

Instituto Nacional de Estadística y Geografía (INEGI). (2019). Disponibilidad y uso de TIC. https://www.inegi.org.mx/temas/ticshogares/.

Instituto Nacional para la Evaluación de la Educación (INEE). (2019). Principales cifras nacionales. Educación Básica y Media Superior Inicio del ciclo escolar 2016-2017. INEE. https://www.inee.edu.mx/wp-content/up loads/2019/08/P2M111.pdf

Jayasankara, K., Menon, K. R., \& Thattil, A. (2018). Academic stress and its sources among university students. Biomedical Pharmacology Journal, 11(1), 531-537. https://dx.doi.org/10.13005/bpj/1404

Jeff, V. (2020). Consideraciones sobre la salud mental en la pandemia de Covid-19. Revista Peruana de Medicina Experimental y Salud Pública, 37(2), 1327. https://doi.org/10.17843/rpmesp.2020.372.5419.

Jerez, M., \& Oyarzo, C. (2015). Estrés académico en estudiantes del Departamento de Salud de la Universidad de Los Lagos Osorno. Revista Chilena de Neuro-Psiquiatría, 53(3), 149-157. http://dx.doi.org/10.4067 /S0717-92272015000300002.

Lazarus, R., \& Folkman, S. (1986). Estrés y procesos cognitivos. Martínez Roca. 
Macbani, P., Ruvalcaba, J., Vásquez, P., Ramírez, A., González, K., Arredondo, K., Monroy, K., Tandehuitl, N., García, M., \& Trejo, A. (2018). Estrés académico, estresores y afrontamiento en estudiantes de odontología en el Centro de Estudios Universitarios Metropolitano Hidalgo (CEUMH). Journal of Negative \& Positive Results, 3(7), 522-530. https://doi.org/10 .19230/jonnpr.2512.

Martín, G., Lucas, B., \& Pulido, R. (2011). Diferencias de género en el afrontamiento en la adolescencia. BROCAR, 35, 157-166. https://doi.or $\mathrm{g} / 10.18172 /$ brocar. 1599

Méndez, C. F.X., Olivares, R. J., \& Quiles, S. M.J. (2014). Técnicas de relajación y respiración. En R. J. Olivares \& C. F. X. Méndez (Eds.), Técnicas de modificación de conducta (p. 27-77). Editorial Biblioteca Nueva.

Montesó, C. P. (2014). La construcción de los roles de género y su relación con el estrés crónico y la depresión en las mujeres. Comunitaria: International Journal of Social Work and Social Sciences, 8, 105-126. https://doi.org/10 $.5944 /$ comunitania.8.6

Niño, S., Castellanos, J., \& Patrón, F. (2021). Contraste de experiencias de estudiantes universitarios en dos escenarios educativos: enseñanza en línea vs. Enseñanza remota de emergencia. Revista de Educación a Distancia, 21(65), 7. https://doi.org/10.6018/red.440731.

Olivares, R., J., Méndez C. F. X., \& Lozano, B. M. (2014). Técnicas de autocontrol. En R. J. Olivares \& C. F. X. Méndez (Eds.), Técnicas de modificación de conducta (pp. 371-408). Editorial Biblioteca Nueva.

Ozamiz, N., Dosil, M., Picaza, M., \& Idoiaga, N. (2020). Niveles de estrés, ansiedad y depresión en la primera fase del brote del COVID-19 en una muestra recogida en el norte de España. Cadernos de Saúde Pública, 36(4), e00054020. https://doi.org/10.1590/0102-311X00054020.

Piemontesi, S., Heredía, D., Furlan, L., Sánchez, J., \& Martínez, M. (2012). Ansiedad ante los exámenes y estilos de afrontamiento ante el estrés académico en estudiantes universitarios. Anales de Psicología, 28(1), 89-96.

Portillo Peñuelas, S. A., Castellanos Pierra, L. I., Reynoso González, Ó. U., \& Gavotto Nogales, O. I. (2020). Enseñanza remota de emergencia ante la pandemia Covid-19 en Educación Media Superior y Educación Superior. Propósitos y Representaciones, 8(SPE3), e589. http://dx.doi.org/10.20511 /pyr2020.v8nSPE3.589

Programa de Naciones Unidas para el Desarrollo. (2020). Desarrollo humano y COVID-19 en México: desafios para una recuperación sostenible. UNDP. https://www.mx.undp.org/content/mexico/es/home/library/p overty/desarrollo-humano-y-covid-19-en-mexico-.html.

Quezada, B., \& González, M. (2012). El papel de los estresores y las condiciones del estado civil para explicar la depresión y el estrés en adolescentes gestantes. Psicología desde el Caribe, 29(1), 19-46.

Reverter Bañón, S. (2003). La perspectiva de género en la filosofía. Feminismo/ s, I(Jun), 33-50. http://dx.doi.org/10.14198/fem.2003.1.04

Reyes, V., Reséndiz, A., Alcázar, R., \& Reidl, L. (2017). Las estrategias de afrontamiento que utilizan los adolescentes ante situaciones que provocan miedo. Psicogente, 20(38), 240-255. http://doi.org/10.17081/psico.20.3 8.2544

Rodríguez, B., González, P., \& Blanco, L. (2014). Estresores académicos percibidos por estudiantes pertenecientes a la escuela de enfermería de 
Ávila, centro adscrito a la Universidad de Salamanca. Revista Enfermeria Castilla y León, 6(2), 98-105.

Román, C., Ortiz, F., \& Hernández, Y. (2008). El estrés académico en estudiantes latinoamericanos de la carrera de medicina. Revista Iberoamericana de Educación, 46, 7-25. https://doi.org/10.35362/rie467 1911.

Secretaría de Gobernación. (2013). Diario Oficial de la Federación. http://dof.g ob.mx/nota_detalle.php?codigo $=5284148 \&$ fecha $=04 / 01 / 2013$.

Segura, R., \& Pérez, I. (2016). Impacto diferencial del estrés entre hombres y mujeres: una aproximación desde el género. Alternativas en Psicología, 36, 105-120.

Silva Ramos, M. F., López Cocotle, J. J., Sánchez de la Cruz, O., \& González Angulo, P. (2019). Estrés académico en estudiantes de Licenciatura en Enfermería. Revista Cientifica de Enfermería, 18, 25-39.

Sociedad Mexicana de Psicología. (2007). Código ético del psicólogo. Editorial Trillas.

Toribio, C., \& Franco, S. (2016). Estrés académico: el enemigo silencioso del estudiante. Salud y Administración, 3(7), 11-18.

Vizoso Gómez, C. M., \& Arias Gundín, O. (2016). Estresores académicos percibidos por estudiantes universitarios y su relación con el burnout y el rendimiento académicos. Anuario de Psicología, 46(2), 90-97. https://do i.org/10.1016/j.anpsic.2016.07.006

World Economic Forum. (2019). The global competitiveness report 2019. http:/ /www.cdi.org.pe/InformeGlobaldeCompetitividad/. 